\title{
Accuracy of Anti-GBM Antibodies in Diagnosing Anti-Glomerular Basement Membrane Disease: A Systematic Review and Meta-Analysis
}

\author{
Akihiro Shiroshita $^{a}$ b Yasuhiro Oda ${ }^{c}$ Seiji Takenouchid ${ }^{d}$ Noboru Hagino $^{e}$ \\ Yuki Kataoka ${ }^{b, f, g}$

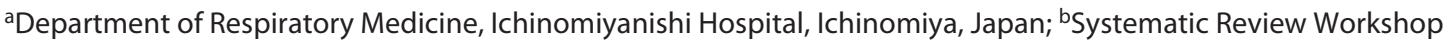 \\ Peer Support Group (SRWS-PSG), Osaka, Japan; 'Division of Nephrology and Endocrinology, the University of Tokyo \\ Graduate School of Medicine, Tokyo, Japan; dDepartment of Rheumatology, Ichinomiyanishi Hospital, Ichinomiya, \\ Japan; 'Department of Rheumatology, Teikyo University Chiba Medical Center, Chiba, Japan; fDepartment of \\ Respiratory Medicine, Hyogo Prefectural Amagasaki General Medical Center, Amagasaki, Japan; 9Department of \\ Hospital Care Research Unit, Hyogo Prefectural Amagasaki General Medical Center, Amagasaki, Japan
}

\section{Keywords}

Anti-glomerular basement membrane disease .

Autoantibody · Diagnostic accuracy · Meta-analysis ·

Systematic review

\begin{abstract}
Background: The sensitivity and specificity of anti-glomerular basement membrane (GBM) antibodies have not been systematically analyzed. In this systematic review, we aimed to evaluate the diagnostic accuracy of anti-GBM antibodies for anti-GBM disease. Summary: Potential studies were searched using MEDLINE, Embase, the Cochrane Library, and the International Clinical Trials Registry Platform based on the index test and target condition. The inclusion criteria were prospective or retrospective cohort studies or casecontrol studies assessing the sensitivity and specificity of anti-GBM antibodies, and the reference standard was clinical diagnosis including biopsy results. The exclusion criteria were review articles, case reports, animal studies, and in vitro studies. Quality assessment was conducted based on the Quality Assessment of Diagnostic Accuracy Studies-2. The pooled estimates of sensitivity and specificity were calculated using a bivariate random-effects model. The overall qual-
\end{abstract}

ity was evaluated using the Grades of Recommendation, Assessment, Development, and Evaluation. Six studies (1,691 patients) and 11 index tests were included in our systematic review. A high risk of bias and concerns regarding the applicability of patient selection were noted because of the casecontrol design in $67 \%$ of the included studies. The pooled sensitivity and specificity were $93 \%(95 \% \mathrm{Cl}: 84-97 \%)$ and 97\% (95\% Cl: 94-99\%), respectively. The certainty of evidence was low because of the high risk of bias and indirectness. Key Messages: Anti-GBM antibodies may exhibit high sensitivity and specificity in the diagnosis of anti-GBM disease. Further cohort studies are needed to confirm their precise diagnostic accuracy and compare diagnostic accuracies among different immunoassays.

(c) 2021 S. Karger AG, Basel

\section{Introduction}

Anti-glomerular basement membrane (GBM) disease is characterized by glomerulonephritis and pulmonary hemorrhage caused by anti-GBM antibodies or autoantibodies against the key epitope regions of the non-collagenous domain 1 of the $\alpha 3$-chain of type IV collagen [1]. 
Anti-GBM disease accounts for $10 \%$ of cases of rapidly progressive glomerulonephritis, and it can be fatal without aggressive treatments [2]. Serological tests have been widely used in clinical settings with the following techniques: radioimmunoassay, fluorescence enzyme immunoassay, enzyme-linked immunosorbent assay (ELISA), Western blotting, chemiluminescent enzyme immunoassay, and multiplex immunoassay. Nevertheless, no systematic review has evaluated the sensitivity and specificity of anti-GBM antibodies in the diagnosis of anti-GBM disease. This clinical question is important, especially because a kidney biopsy cannot always be performed in patients with a fulminant course of anti-GBM disease [3]. All considered, this systematic review was conducted to evaluate the diagnostic accuracy of serum anti-GBM antibodies for detecting anti-GBM disease among patients suspected to have anti-GBM disease in tertiary care centers.

\section{Material and Methods}

\section{Study Design}

The research protocol of our systematic review was registered before the screening commenced at the International Prospective Register of Systematic Reviews (protocol registration No: CRD42021227832) [4]. This review adheres to the preferred reporting items for systematic reviews and meta-analyses: the PRISMA statement [5]. The PRISMA checklist is uploaded as the online suppl. Table S1 (see www.karger.com/doi/10.1159/000518362 for all online suppl. material). Informed consent was not required owing to the secondary data analysis design.

\section{Search Strategy and Study Selection}

We searched the Medical Literature Analysis and Retrieval System Online, Excerpta Medica Database, Cochrane Library, and the International Clinical Trials Registry Platform using search terms related to "anti-glomerular basement membrane antibody" and "anti-glomerular basement membrane disease" without restricting language or dates (online suppl. Table 2). Additional articles were searched by reviewing all reference lists of the included articles and using the Web of Science. The inclusion criteria were prospective or retrospective cohort studies or case-control studies meeting the following requirements: (a) the sensitivity and specificity of antiGBM antibodies (fluorescence enzyme immunoassay, ELISA, Western blotting, chemiluminescent enzyme immunoassay, and multiplex immunoassay) were assessed for detecting anti-GBM disease; (b) the reference standard was a clinical diagnosis of antiGBM disease including kidney or lung biopsy. The exclusion criteria were review articles, case reports, and animal studies. Studies detecting anti-GBM antibodies using indirect immunofluorescence were ineligible for our study.

Data Extraction and Quality Assessment

Study selection was performed independently by A.S. and Y.O. After reviewing the titles and abstracts, we identified eligible studies based on their full text. A.S. extracted the following data from the included articles: study characteristics (title, author, country, design, and year of publication), population characteristics (total number of patients, age, and sex), index test information, reference standard information, number of true positives, false positives, true negatives, and false negatives. When only the receiver operating characteristics curve was reported, WebPlotDigitizer 4.4 was used to extract information on sensitivity and specificity [6]. After the data extraction, the risk of bias and concerns for applicability in the included studies were assessed by A.S. and S.T. independently using the Quality Assessment of Diagnostic Accuracy Studies (online suppl. Table 3) [7]. The signaling questions were framed before initiating the review. When reviewers found insufficient data, they contacted the corresponding authors via e-mail. During the entire review process, any disagreement between the reviewers was resolved by discussion.

\section{Statistical Analysis}

Univariate forest plots for sensitivity and specificity of the included studies were generated. The pooled estimates of sensitivity and specificity were calculated using a bivariate random-effects model, accounting for the negative correlation between sensitivity and specificity. The variation in the cutoff values of sensitivity and specificity was considered, and the hierarchical summary receiver operating characteristic curve was created using the parameters estimated with the bivariate random-effects model $[8,9]$. For ad hoc subgroup analysis, the pooled sensitivity and specificity of ELISA were calculated. For statistical analysis, R 4.0.2 (R Foundation for Statistical Computing, Vienna, Austria) and RevMan v5.4 (The Nordic Cochrane Centre, The Cochrane Collaboration, Copenhagen, Denmark) were used $[10,11]$. The R package "mada version 0.5 .10 " was used to calculate the estimates [12]. The overall quality of the study was assessed using the Grades of Recommendation, Assessment, Development, and Evaluation (GRADE) [13]. A.S. and S.T. assessed the quality based on a discussion using the GRADEpro GDT [14].

\section{Results}

The study selection process is illustrated in Figure 1. After title, abstract, and full-text screening, 6 studies were included [3, 15-19]. Eleven index tests were used in the 6 studies. In total, 1,691 patients were included. The study characteristics are summarized in Table 1. Patient demographic characteristics, such as age and sex, could not be collected for the included studies. Among the 6 included studies, 4 (67\%) were designed as case-control studies. Information about the index tests and reference standards is summarized in Table 2. ELISA was the most commonly used test (55\%). For half of the included studies, we could not obtain information on whether immunofluorescence was used for pathological results.

The quality assessments in each index test are summarized in Figure 2; online suppl. Figure 1. During the process, the signaling questions framed initially were 


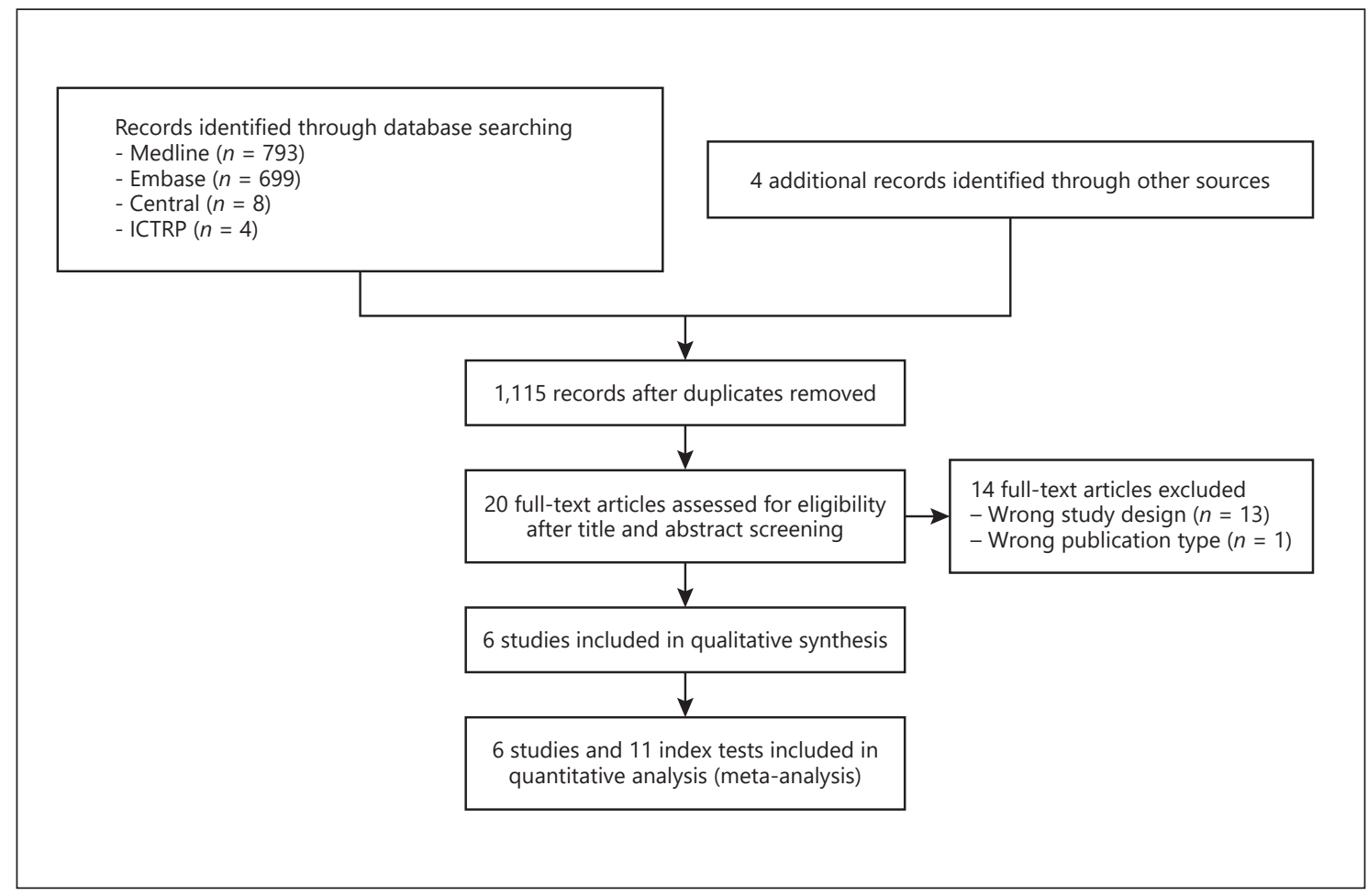

Fig. 1. PRISMA flow chart. The excluded studies after full-text review. Wrong study design: Amiri et al. [29], Fish et al. [30], Jaskowski et al. [31], Li et al. [23], Mahieu et al. [32], Saxena et al. [33], Shen et al. [34], Sommarin et al. [35], Tan et al. [26], Vavilapalli et al. [36], Watad et al. [37], Westman et al. [38], and Xin et al. [39]. Wrong publication type: Lan et al. [40].

Table 1. Characteristics of the included studies

\begin{tabular}{|c|c|c|c|c|}
\hline Study & Country & Design & Setting & Participants, $n$ \\
\hline Rutger et al. [15] & The Netherlands & Case-control & University Hospital & $\begin{array}{l}116 \text { patients ( } 9 \text { anti-GBM disease, } 73 \text { pauci-immune crescentic } \\
\text { glomerulonephritis, } 10 \text { lupus nephritis, } 11 \text { high rheumatoid factor, } \\
8 \text { high IgG, and } 5 \text { healthy controls) }\end{array}$ \\
\hline Sinico et al. [3] & Italy & Case-control & $\begin{array}{l}\text { Acute General } \\
\text { Hospital }\end{array}$ & $\begin{array}{l}52-58 \text { patients (19-22 anti-GBM disease, } 22 \text { granulomatous with } \\
\text { polyangiitis, } 15 \text { microscopic polyangiitis, } 1 \text { eosinophilic granulomatosis } \\
\text { with polyangiitis, } 2 \text { lupus, } 1 \text { idiopathic pulmonary fibrosis, and } 28 \text { blood } \\
\text { donors) }\end{array}$ \\
\hline $\begin{array}{l}\text { Damoiseaux et al. } \\
{[16]}\end{array}$ & The Netherlands & Case-control & & $\begin{array}{l}9 \text { Goodpasture's syndrome, } 87 \text { patients with pauci-immune necrotizing } \\
\text { crescentic glomerulonephritis, } 12 \text { lupus, } 11 \text { positive rheumatoid factor, } \\
9 \text { high IgG, and } 40 \text { healthy controls }\end{array}$ \\
\hline de Joode et al. [17] & The Netherlands & $\begin{array}{l}\text { Retrospective } \\
\text { cohort }\end{array}$ & University Hospital & $\begin{array}{l}260 \text { patients whose samples were sent to the university laboratory for } \\
\text { analysis of ANCA and anti-GBM }\end{array}$ \\
\hline Sowa et al. [18] & Italy & Case-control & $\begin{array}{l}\text { Acute General } \\
\text { Hospital }\end{array}$ & $\begin{array}{l}287 \text { patients ( } 43 \text { anti-GBM disease, } 40 \text { granulomatous with polyangiitis, } \\
2 \text { eosinophilic granulomatosis with polyangiitis, } 42 \text { lupus, } 57 \text { infectious } \\
\text { diseases, and } 55 \text { healthy subjects) }\end{array}$ \\
\hline Busch et al. [19] & The Netherlands & Case-control & University Hospital & 80 patients ( 19 anti-GBM disease, and 51 non-ANCA related vasculitides \\
\hline
\end{tabular}


Table 2. Detailed information about index tests and reference standard in each study

\begin{tabular}{ll}
\hline Study & Index test \\
\hline Rutger et al. [15] & $\begin{array}{l}\text { ANCA-GBM dot blot (biomedical diagnostics, Brugge, Belgium, antigen of } \\
\text { the NC1 domain of type IV collagen in bovine kidney, normal value } \leq 10\end{array}$
\end{tabular}
arbitrary units)

Sinico et al. [3]

- Direct ELISA (anti-GBM Immunoscan, Euro-Diagnostician, Malmö, Sweden, antigen of the M2 subunit from the non-collagenous domain of type IV collagen, normal value $\leq 15$ arbitrary units)

- Direct ELISA (anti-GBM antibodies Wielisa-kit from Wieslab, Lund, Sweden, extracted purified human $\alpha 3$ chain of type IV collagen, normal value $\leq 10$ arbitrary units)

- Direct ELISA (Varelisa GBM antibodies from Pharmacia Diagnostics, Freiburg, Germany, antigen of a human recombinant $\alpha 3$ chain of collagen type IV expressed in insect cells (SF9/baculovirus), normal value $\leq 7$ arbitrary units)

- Direct ELISA (EliA GBM antibodies from Pharmacia Diagnostics, Freiburg, Germany, antigen of a human recombinant $\alpha 3$ chain of collagen type IV expressed in insect cells (SF9/baculovirus), normal value $\leq 8$ arbitrary units)

Damoiseaux et al. Multiplex immunoassay (FIDIS vasculitis, normal value $<25$ arbitrary units) [16]

de Joode et al. [17] - Direct ELISA (The Netherlands)

- Direct ELISA (Phadia ELiA, Thermo Fisher Scientific, Germany, human recombinant antigen)

- ANCA-GBM dot blot (MBG Fot, Biomedical Diagnostics, Belgium)

Sowa et al. [18] Direct ELISA (Phadia [Uppsala/Sweden], Euro Diagnostica [Lundavägen/ Sweden] and GA Generic Assays GmnH [Dahlewitz/Berlin/Germany])

Busch et al. [19] Immunoblot (the EUROLINE MPO/PR3/GBM profile immunoblot, EUROIMMUN AG, Lübeck, Germany, antigen of $\alpha 3$ chains of NC1 domain of type IV collagen in bovine kidney, signal intensity $>5$ )
Reference standards

Focal or diffuse segmental crescentic glomerulonephritis and the presence of linear fluorescence of IgG along the glomerular basement membrane in the kidney biopsy and/or by the presence of specific circulating anti-GBM antibodies

Clinical picture of rapidly progressive glomerulonephritis and the presence of linear fluorescence of $\operatorname{IgG}$ along the glomerular basement membrane in the kidney biopsy
Presence of a glomerular basement membrane $<260$ $\mathrm{nm}$ with a unimodal distribution using the orthogonal intercept method and the absence of IgA deposits by immunohistochemistry; absence of extrarenal disease, and the presence of "Goodpasture-type antigen" using a monoclonal antibody against the collagen part of the a5 chain of type IV collagen on fresh frozen sections

Clinical diagnosis, including kidney biopsy (unclear about immunofluorescence techniques)

Clinical diagnosis, including kidney biopsy (unclear about immunofluorescence techniques)

Clinical diagnosis, including the presence of anti-GBM antibodies, and kidney biopsy (unclear about immunofluorescence techniques)

GBM, glomerular basement membrane; ELISA, enzyme-linked immunosorbent assay.

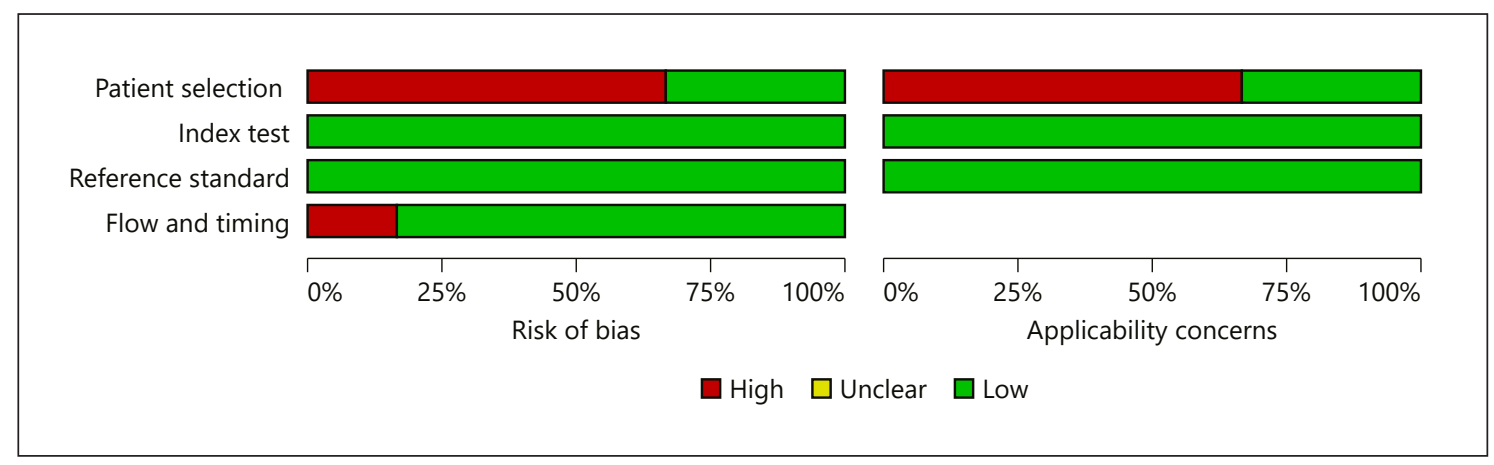

Fig. 2. Assessment of risk of bias and applicability in the included studies. The high risk of bias and high concerns for applicability in the patient selection domain were due to the case-control design. Because the number of excluded patients in the analysis was not clear in Busch et al., we assessed that this study has a high risk of bias in the flow and timing domains. 
not changed. A.S. and S.T. independently evaluated each study and made decisions about the risk of bias and applicability based on a one-by-one discussion. Our assessment of a "high risk of bias" was because of the twostage patient selection, that is, sensitivity was calculated among controls with a non-validated selection process, and specificity was calculated among the anti-GBM groups. In most included studies, it was unclear whether the cutoff value of anti-GBM antibodies was prespecified or the interpretation of kidney biopsy was blinded. We could not obtain detailed information from the corresponding authors of the included studies, and we assessed these studies to have a "low risk of bias" because the researchers followed the product insert of the antiGBM antibodies and pathological tests, which are objective in nature.

Univariate forest plots revealed little variability in sensitivity and specificity among the studies (online suppl. Fig. 2). The meta-analysis of 11 index tests revealed that the pooled estimates of sensitivity and specificity were 93\% (95\% CI: 84-97\%) and 97\% (95\% CI: 94-99\%). As presented in Figure 3, the 95\% confidence region showed high sensitivity and specificity after considering the heterogeneity of the cutoff points. Because of the small sample size, the $95 \%$ prediction region showed a wide range of sensitivities and specificities. Our post-hoc subgroup analysis revealed that among ELISAs, the pooled sensitivity and specificity were $94 \%$ (95\% CI: 87-97\%) and 97\% (92-99\%), respectively. We simulated the change in diagnostic accuracy based on the prevalence rates (Table 3); it showed a low false-negative rate and high true positive rate. The certainty of evidence was low because of serious concerns regarding the risk of bias and applicability based on case-control designs.

\section{Discussion}

In this systematic review, we aimed to evaluate the diagnostic accuracy of anti-GBM antibodies in patients suspected with anti-GBM disease. Based on our study, antiGBM antibodies, especially detected using ELISA, had extremely high sensitivity and specificity. The certainty of evidence was low due to the 2 -stage patient selection process.

One of the key findings of our systematic review was that the low false-negative rate might be sufficient to rule out anti-GBM disease in the primary assessment while awaiting biopsy results. Anti-GBM is suspected in patients with rapidly progressive glomerulonephritis and/

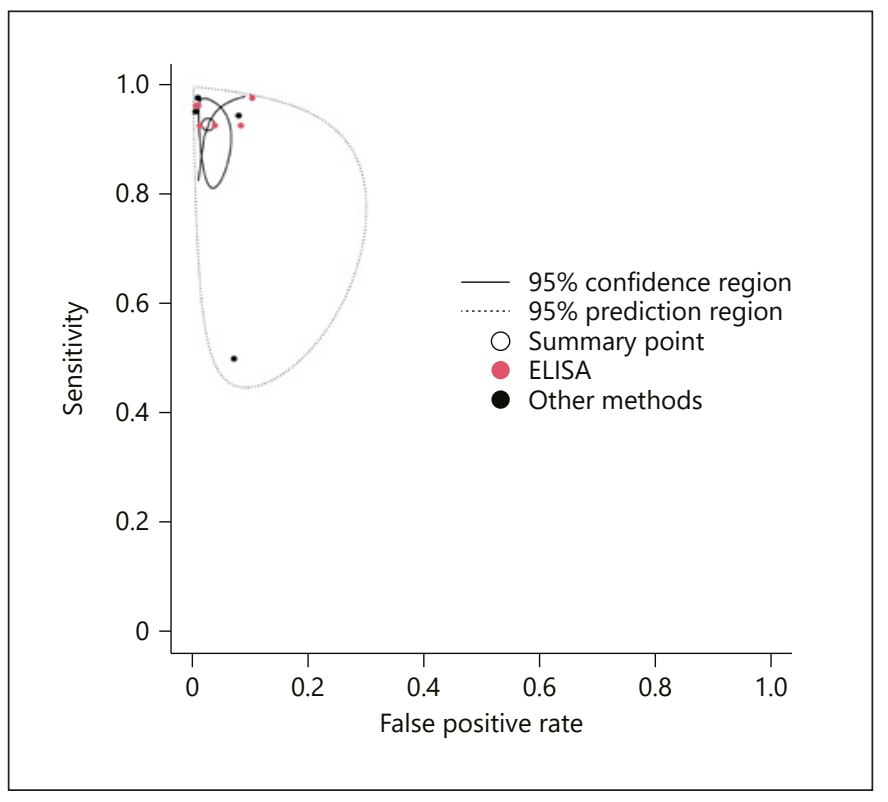

Fig. 3. Hierarchical summary of receiver operating characteristics curve of serum anti-GBM antibodies. The pooled estimate of sensitivity was 93\% (95\% CI: 84-97\%), and the pooled specificity was 97\% (95\% CI: 94-99\%). Although the 95\% prediction region was wide because of the small sample size, the $95 \%$ confidence region revealed high sensitivity and specificity. GBM, glomerular basement membrane.

or pulmonary hemorrhage. Based on the $10 \%$ prevalence of anti-GBM disease among patients with rapidly progressive glomerulonephritis, the false-negative rate was low [2]. Because other differential diagnoses of rapidly progressive glomerulonephritis would require a kidney biopsy, physicians cannot spare biopsies based on a negative result of anti-GBM antibodies. However, patients might not be treated with specific treatments for antiGBM disease, such as plasmapheresis, while awaiting biopsy results [20].

Another key finding was the extremely high true positive rate of anti-GBM antibodies, which has 3 important clinical implications. The meaning of high specificity of anti-GBM antibodies might vary depending on the timing and waiting time of anti-GBM antibodies and kidney biopsy. First, patients with a positive test result and a high risk of complications in kidney biopsy procedure may be reasonably diagnosed with anti-GBM disease without performing kidney biopsy and be reasonably started with specific treatments for anti-GBM disease. An examination of anti-GBM antibodies, accompanied by blood sampling, is only slightly invasive, whereas kidney biopsy can cause serious complications. Second, physicians could 


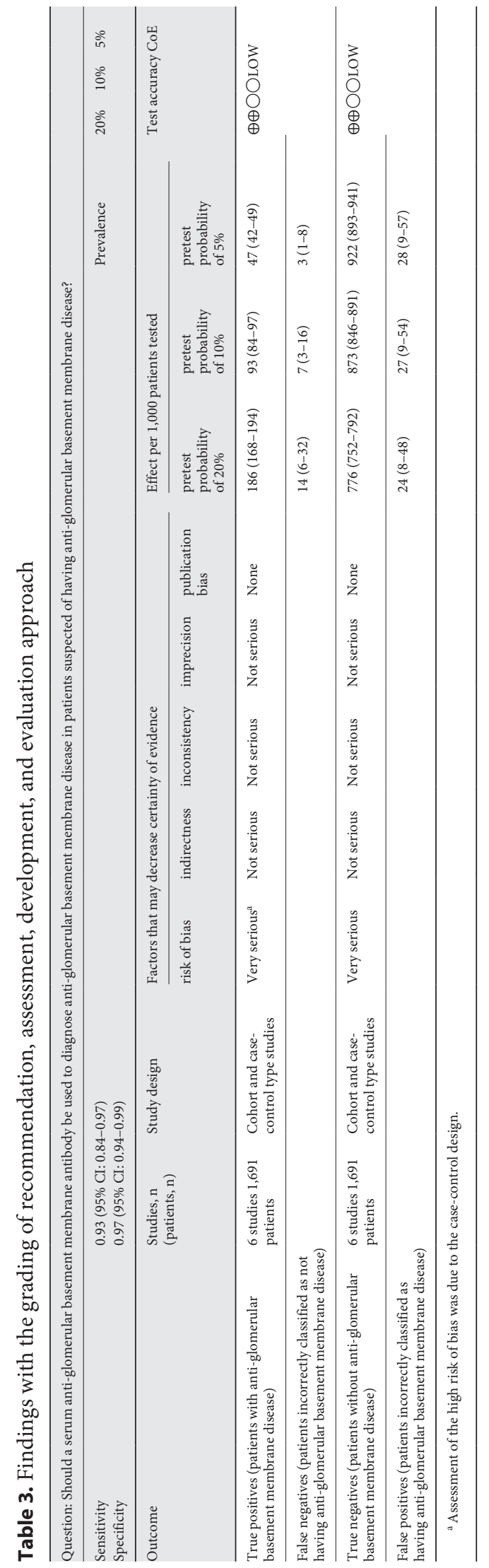

rule in anti-GBM disease before receiving the result of kidney biopsy and start specific treatments for anti-GBM disease. Following early treatments, the survival rate has substantially improved [21]. Third, when patients with end-stage kidney disease receive a positive result for antiGBM antibodies and do not have any specific findings associated with other differential diagnoses, it might motivate physicians to avoid immunosuppressive therapy and plasmapheresis because end-stage kidney disease caused by anti-GBM disease can be irreversible [22, 23].

In our systematic review, although the sensitivity and specificity of ELISA were quite high, we could not conclude as to which methodology was the most accurate. Among the excluded studies, there was a comparison among different methodologies [24-26]. Although these studies were excluded because the reference standards were not kidney biopsies, in these studies, chemiluminescence immunoassay and ELISA were associated with high sensitivity and specificity, similar to the results of our systematic review. Further head-to-head comparison studies are needed to detect the differences among the methodologies.

\section{Conclusion}

The low certainty of evidence limits our confidence in concluding on our clinical question. Although we assessed "low risk of bias" in the index test and reference standard domains, a high risk of bias in the domain of patient selection was evident. In clinical settings, acute nephritic syndrome (acute kidney injury, hematuria, and proteinuria) and/or pulmonary hemorrhage are the main manifestations [27]. The diagnostic accuracy for these patients was relevant. In future studies, we recommend a prospective or retrospective cohort study design to avoid patient selection bias.

Our systematic review had several limitations. First, the number of included studies was small. As the $95 \%$ prediction region was wide, we are not sure that future studies will show high sensitivity and specificity. Multiple index tests were included in the same studies. Because of the same study participants, the diagnostic accuracy could be overestimated. Furthermore, in this systematic review, we could not elucidate the complex mechanism of anti-GBM antibody in the blood and kidney [28]. Additional studies are needed from the perspective of both clinical and basic medicine. Second, we could not obtain information on how the cutoff value was determined and what was the antigenic substrate in most of the included 
studies. Commercial kits were used in the studies, and thus, we assumed that there would be a cutoff value determined by the company or laboratory. Third, different cutoff values were used in the studies. Although the heterogeneity of cutoff values was considered in the statisti$\mathrm{cal}$ analysis, they should be standardized in the future. It is also of note that this meta-analysis focused only on the positive/negative judgments based on the cutoff values, while the magnitude of antibody titers may affect decisions in real-world clinical practice. Finally, we could not obtain detailed information on whether or not immunofluorescence was used for the pathological diagnosis of anti-GBM disease in half of the included studies. Although the diagnostic accuracy of immunofluorescence assay is not perfect, it has been used in daily practice. All of the included studies diagnosed anti-GBM disease based on both the pathological results and clinical course, and therefore misclassification of anti-GBM could be minimized. Nevertheless, some misclassifications may have occurred. Physicians should be aware that the diagnostic accuracy of immunofluorescence assay is not perfect.

In conclusion, anti-GBM antibodies may have high sensitivity and specificity when diagnosing anti-GBM disease among patients suspected of having this disease. Further cohort studies are needed to precisely evaluate the diagnostic accuracy and compare diagnostic accuracies among different assays.

\section{Acknowledgments}

The authors would like to thank Dr. Megha Uppin from the Nizam's Institute of Medical Sciences, Dr. Renato Sinico from the Università degli Studi di Milano, and Dr. Jan Damoiseaux from the Maastricht University Medical Center for providing us with the detailed information necessary for this study.

\section{Conflict of Interest Statement}

The authors have no conflicts of interest to declare.

\section{Funding Sources}

This study was funded by the Systematic Review Workshop Peer Support Group (SRWS-PSG: https://community.camp-fire. $\mathrm{jp} /$ projects/view/187310). This funder played no role in the study design, study execution, data analyses, data interpretation, or decision to submit the results.

\section{Author Contributions}

A.S., Y.O., S.T., N.H. and Y.K. contributed to the conception and design of the work. A.S. and O.Y. contributed to the acquisition of data. A.S. and Y.K. contributed to analysis. A.S., Y.O., N.H. and Y.K. contributed to the interpretation of data. A.S., O.Y., and Y.K. wrote a draft of the manuscript. All authors revised the manuscript critically and approved the final version of the manuscript. A.S., Y.O., S.T., N.H. and Y.K. agreed to be accountable for all aspects of the work.

\section{References}

1 Hellmark T, Segelmark M. Diagnosis and classification of Goodpasture's disease (antiGBM). J Autoimmun. 2014 Feb;48-9:108-12.

2 Andrassy K, Küster S, Waldherr R, Ritz E. Rapidly progressive glomerulonephritis: analysis of prevalence and clinical course. Nephron. 1991;59(2):206-12.

3 Sinico RA, Radice A, Corace C, Sabadini E, Bollini B. Anti-glomerular basement membrane antibodies in the diagnosis of Goodpasture syndrome: a comparison of different assays. Nephrol Dial Transplant. 2006 Feb; 21(2):397-401.

4 The international prospective register of systematic reviews [Internet]. [cited 2021 Jan 29]. Available from: https://www.crd.york. ac.uk/prospero/.

5 McInnes MDF, Moher D, Thombs BD, McGrath TA, Bossuyt PM, Clifford T, et al. Preferred reporting items for a systematic review and meta-analysis of diagnostic test accuracy studies: the PRISMA-DTA statement. JAMA. 2018;319(4):388-96.

6 Rohatgi A. WebPlotDigitizer-copyright 2010-2020 [Internet]; 2020. [cited $2021 \mathrm{Feb}$ 18]. Available from: https://apps.automeris. io/wpd/.
7 Whiting PF, Rutjes AW, Westwood ME, Mallett S, Deeks JJ, Reitsma JB, et al. QUADAS-2: a revised tool for the quality assessment of diagnostic accuracy studies. Ann Intern Med. 2011;155:529-36.

8 Arends LR, Hamza TH, van Houwelingen JC, Heijenbrok-Kal MH, Hunink MG, Stijnen T. Bivariate random effects meta-analysis of ROC curves. Med Decis Making. 2008 Jun; 28(5):621-38.

9 Chu H, Cole SR. Bivariate meta-analysis of sensitivity and specificity with sparse data: a generalized linear mixed model approach. J Clin Epidemiol. 2006 Dec;59(12):1331-3; author reply 1332-33.

10 R: the R project for statistical computing [Internet]. [cited $2021 \mathrm{Feb}$ 18]. Available from: https://www.r-project.org/.

11 RevMan [Internet]. [cited 2021 Feb 18]. Available from: online-learning/core-software-cochrane-reviews/revman.

12 Doebler P. mada: meta-analysis of diagnostic accuracy. 2020. Available from: https:// CRAN.R-project.org/package $=$ mada.

13 Balshem $H$, Helfand $M$, Schünemann HJ, Oxman AD, Kunz R, Brozek J, et al. GRADE guidelines: 3 . Rating the quality of evi- dence. J Clin Epidemiol. 2011 Apr;64(4): 401-6.

14 GRADEpro [Internet]. [cited $2021 \mathrm{Feb}$ 18]. Available from: https://gradepro.org/.

15 Rutgers A, Damoiseaux J, Roozendaal C, Limburg PC, Stegeman CA, Tervaert JW. ANCAGBM Dot-Blot: evaluation of an assay in the differential diagnosis of patients presenting with rapidly progressive glomerulonephritis. J Clin Immunol. 2004 Jul;24:435-40.

16 Damoiseaux J, Vaessen M, Knapen Y, Csernok E, Stegeman CA, Van Paassen P, et al. Evaluation of the FIDIS vasculitis multiplex immunoassay for diagnosis and follow-up of ANCA-associated vasculitis and Goodpasture's disease. Ann N Y Acad Sci. 2007 Aug; 1109(1):454-63.

17 de Joode AA, Roozendaal C, van der Leij MJ, Bungener LB, Sanders JS, Stegeman CA. Performance of two strategies for urgent ANCA and anti-GBM analysis in vasculitis. Eur J Intern Med. 2014 Feb;25(2):182-6.

18 Sowa M, Trezzi B, Hiemann R, Schierack P, Grossmann K, Scholz J, et al. Simultaneous comprehensive multiplex autoantibody analysis for rapidly progressive glomerulonephritis. Medicine. 2016 Nov;95(44):e5225.
Role of Anti-Glomerular Basement Membrane Antibodies 
19 Busch MH, Aendekerk JP, van Beers JJBC, van Paassen P, Damoiseaux JGMC. Evaluation of the diagnostic performance of an immunoblot for ANCA and anti-GBM antibody detection. Autoimmunity. 2021;54(1):45-50.

20 Walsh M, Merkel PA, Peh CA, Szpirt WM, Puéchal X, Fujimoto $S$, et al. Plasma exchange and glucocorticoids in severe ANCA-associated vasculitis. N Engl J Med. 2020 Feb;382: $622-31$.

21 Greco A, Rizzo MI, De Virgilio A, Gallo A, Fusconi M, Pagliuca G, et al. Goodpasture's syndrome: a clinical update. Autoimmun Rev. 2015 Mar;14(3):246-53.

22 Lindic J, Vizjak A, Ferluga D, Kovac D, Aleš A, Kveder R, et al. Clinical outcome of patients with coexistent antineutrophil cytoplasmic antibodies and antibodies against glomerular basement membrane. Ther Apher Dial. 2009 Jul;13(4):278-81.

23 Li FK, Tse KC, Lam MF, Yip TP, Lui SL, Chan GS, et al. Incidence and outcome of antiglomerular basement membrane disease in Chinese. Nephrology. 2004 Apr;9(2):100-4.

24 Litwin CM, Mouritsen CL, Wilfahrt PA, Schroder MC, Hill HR. Anti-glomerular basement membrane disease: role of enzymelinked immunosorbent assays in diagnosis. Biochem Mol Med. 1996 Oct;59(1):52-6.

25 Mahler M, Radice A, Sinico RA, Damoiseaux J, Seaman A, Buckmelter K, et al. Performance evaluation of a novel chemiluminescence assay for detection of anti-GBM antibodies: an international multicenter study. Nephrol Dial Transplant. 2012 Jan;27(1):243-52.

26 Tan Y, Pang W, Jia X, Zhao MH. Comparison of the performance of a chemiluminescence assay and an ELISA for detection of antiGBM antibodies. Ren Fail. 2020;42:48-53.
27 Lazor R, Bigay-Gamé L, Cottin V, Cadranel J, Decaux O, Fellrath JM, et al. Alveolar hemorrhage in anti-basement membrane antibody disease: a series of 28 cases. Medicine. 2007 May;86(3):181-93.

28 Henderson SR, Salama AD. Diagnostic and management challenges in Goodpasture's (anti-glomerular basement membrane) disease. Nephrol Dial Transplant. 2018 Feb; 33(2):196-202.

29 Amiri ML, Trabelsi R, Ounissi M, Chargui S, Jerbi M, Gaied H, et al. P0507 Anti-glomerular basement membrane disease. About 32 cases. Nephrol Dial Transplant. 2020 Jun; 35(Suppl 3).

30 Fish AJ, Kleppel M, Jeraj K, Michael AF. Enzyme immunoassay of anti-glomerular basement membrane antibodies. J Lab Clin Med. 1985 Jun;105(6):700-5.

31 Jaskowski TD, Martins TB, Litwin CM, Hill HR. Comparison of four enzyme immunoassays for the detection of immunoglobulin $G$ antibody against glomerular basement membrane. J Clin Lab Anal. 2002 Apr;16(3):143-5.

32 Mahieu P, Lambert PH, Miescher PA. Detection of anti-glomercular basement membrane antibodies by a radioimmunological technique. Clinical application in human nephropathies. J Clin Invest. 1974 Jul;54:12837.

33 Saxena R, Bygren P, Arvastson B, Wieslander J. Circulating autoantibodies as serological markers in the differential diagnosis of pulmonary renal syndrome. J Intern Med. 1995 Aug;238(2):143-52.
34 Shen CR, Jia XY, Cui Z, Yu XJ, Zhao MH Clinical-pathological features and outcome of atypical anti-glomerular basement membrane disease in a large single cohort. Front Immunol. 2020;11:2035.

35 Sommarin Y, Persson Å, Wieslander J. Rapid vasculitis screening test detecting anti-GBM, capture PR3 ANCA and capture MPO ANCA. APMIS. 2009;117:176.

36 Vavilapalli S, Madireddy N, Uppin MS, Kalidindi K, Gudithi S, Taduri G, et al. Anti-glomerular basement membrane disease: a clinicomorphological study of 16 cases. Indian J Pathol Microbiol. 2020;63(2):226-9.

37 Watad A, Bragazzi NL, Sharif K, Shovman O, Gilburd B, Amital H, et al. Anti-glomerular basement membrane antibody diagnostics in a large cohort tertiary center: should we trust serological findings? Isr Med Assoc J. 2017 Jul;19(7):424-8.

38 Westman KW, Bygren PG, Eilert I, Wiik A, Wieslander J. Rapid screening assay for antiGBM antibody and ANCAs; an important tool for the differential diagnosis of pulmonary renal syndromes. Nephrol Dial Transplant. 1997 Sep;12(9):1863-8.

39 Xin G, Zhao M, Ding J, Wang H. Purification of alpha chain $\mathrm{NCl}$ domains of type IV collagen from bovine kidney and their application in ELISA for detecting anti-glomerular basement membrane antibodies. Beijing Da Xue Xue Bao Yi Xue Ban. 2004 Jan;35(5):4948.

40 Lan P, Xie L. Therapy and outcome of antiglomerular basement membrane disease: a single-center experience. J Am Soc Nephrol. 2019;30:935 